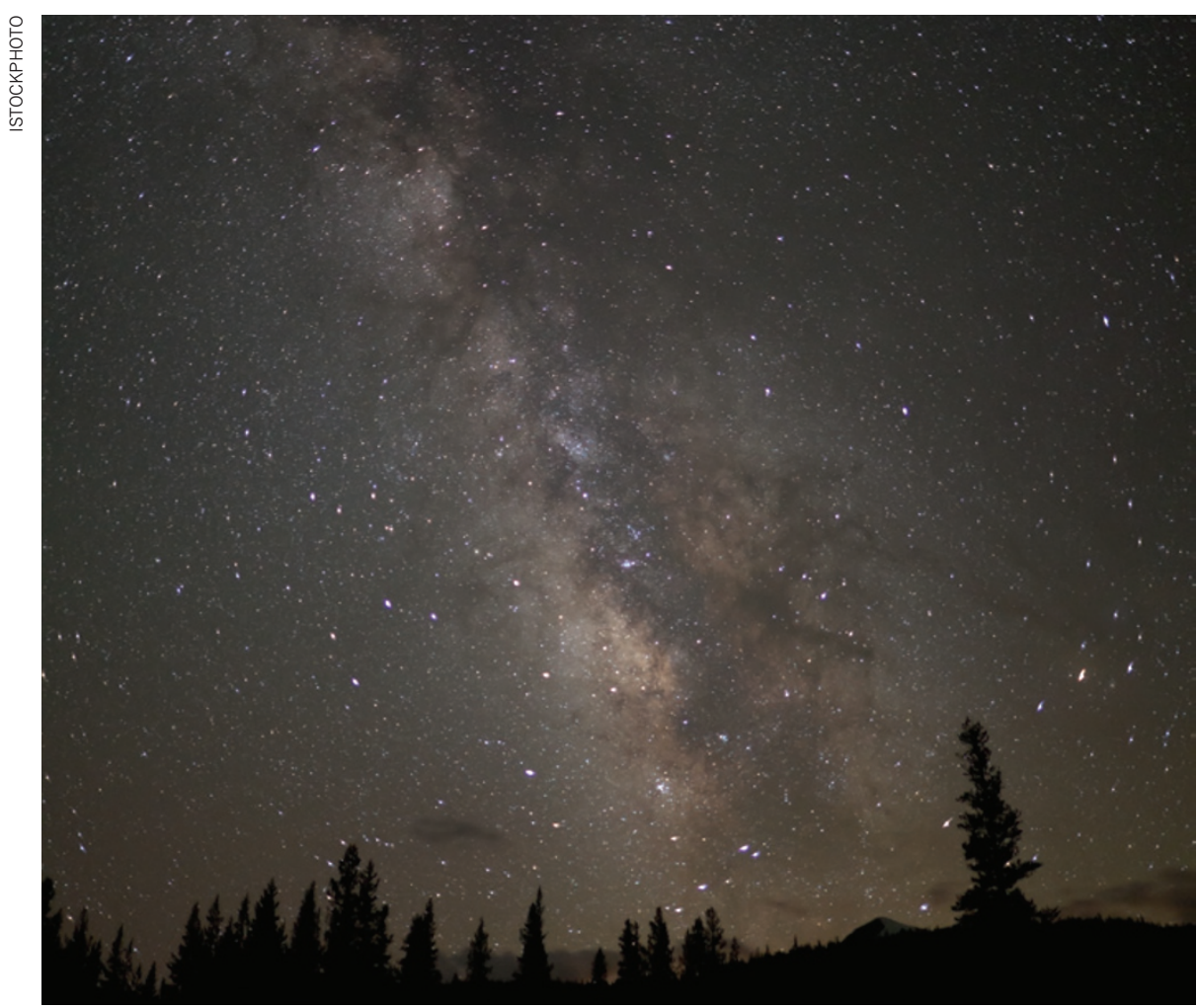

ASTRONOMY

\title{
A conversation about observation
}

In the spirit of the Lindau Meeting, we present a dialogue between a Nobel laureate and a young researcher. This interchange started online, where it continues to unfold. Here is a digest of this conversation, which has developed across time and space.

\section{MEET THE PARTICIPANTS \\ Two astronomers correspond}

John Mather shared the 2006 Nobel

Prize in Physics for his work on the cosmic microwave background radiation. Mather is currently at NASA, focusing on building Hubble's successor, the James Webb Space Telescope (JWST), which he spoke about at the 2012 Lindau Meeting. The JWST is due to launch in 2018 and will orbit the Sun about 1.5 million kilometres further out than the Earth's orbit (in a Lagrange point) to observe the very first galaxies. Minnie Mao recently took a position as a postdoctoral researcher at the National Radio Astronomy Observatory (NRAO) in Socorro, New Mexico - leaving her native Australia. Minnie uses radio and optical telescopes to study the evolution of galaxies to determine how they have formed and evolved since the Big Bang 13.7 billion years ago.

\section{"I'm trying to figure out what my role is in the world of astronomy."}

\section{Dear Professor Mather,}

It was really lovely to meet you at the Lindau Meeting. I had a wonderful, thoughtprovoking week that left me feeling buzzed up and raring to do science!

My name is Minnie and I've just started a postdoc at the NRAO. I submitted my $\mathrm{PhD}$ on the cosmic evolution of radio sources in April and will (hopefully!) graduate from the University of Tasmania in December this year. New Mexico is somewhat different from Tasmania, but I'm really enjoying it here - especially being based at the array operations centre for the Karl G. Jansky Very Large Array (VLA).

This brings me to why I do astronomy: I love observing. I love the process of deciding to 'look' at something, setting up the telescope, waiting in breathless anticipation for the photons to hit the detector, or watching the cross-correlations dance across a computer screen. I even love reducing the data, knowing that shortly I will be seeing something no one has ever seen and using it to answer my science questions.

What do you think will be the most important result to come from the JWST? Will they be driven by predicted theories or by observations that yield completely unexpected results, which we couldn't possibly have known had we not observed something new, as was the case with the Hubble Deep Field?

I love my new job. Learning all about the VLA is so much fun, but now that I'm starting to get used to the fact that I'm no longer a student, I'm trying to figure out what my role is in the world of astronomy.

\section{Clear skies,}

Minnie Yuan Mao

\section{"After all these years, we still have a hard time making a theoretical supernova explode."}

\section{Dear Minnie,}

Thanks for your letter! I like to be called John; I'm still not used to formal titles.

My own passion for physics and astronomy has a lot to do with a desire to build things. When I was a teenager I learned a lot from the Scientific American monthly feature called The Amateur Scientist, and I borrowed the 3-volume book series Amateur Telescope Making from the library over and over again. I did build a small telescope, but 
I didn't have the patience to grind a mirror — I bought that. I also loved thinking about electronics, and I wanted to be a ham radio operator, so I assembled a Heathkit AR-3 radio receiver (with vacuum tubes) and read The ARRL Handbook from cover to cover, over and over.

There's a wonderful book by Martin Harwit called Cosmic Discovery that lists the greatest discoveries in astronomy, and shows what made them possible. In almost every case, the discovery came from new equipment, and in a pretty large fraction of cases the equipment was designed for some other purpose. So I see a path to great discovery: imagine a measurement that could answer a question, and build something to make the measurement. Then, either you answer the question, or you find something new. But in either case, it will be something nobody else could do. Have you given any thought to good strategies for making your own discoveries?

That leads to your other question: what will be the most important discoveries with the JWST? Of course, I don't know specifically, but it seems that surprise is part of importance - if everyone knows something already, it is not a discovery. No theorists or observers predicted the correct story for galaxy formation before the Hubble Space Telescope was launched, neither group predicted cosmic dark energy or dark matter (though Fritz Zwicky saw evidence for dark matter in the 1930s), and neither predicted the nature and abundance of planetary systems beyond our own Solar System. My friends doing numerical simulations of stars and galaxies in supercomputers agree that they are not going to predict what we will find with any certainty - the complexity is just too great. And after all these years, we still have a hard time making a theoretical supernova explode.

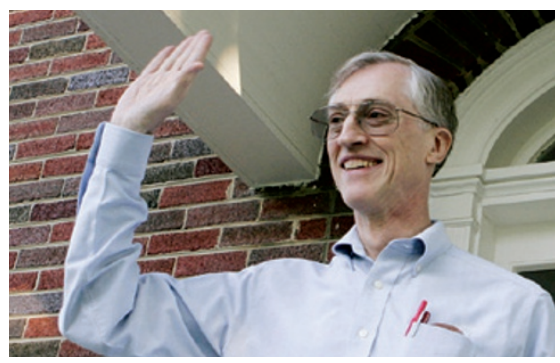

NASA's John Mather says discoveries come from new equipment.

So, I think the JWST will lead to some great surprise that nobody is even thinking about today. Perhaps, you will be the one to find that surprise.

With best wishes for your new role as a Discoverer!

John Mather

\section{"Learning is not just about accumulating information; it's also about the process of getting that information."}

\section{Dear John,}

I like the 'discoverer' tag: I think that is what science is about - the discovery of new things, new phenomena, new concepts, new ideas.

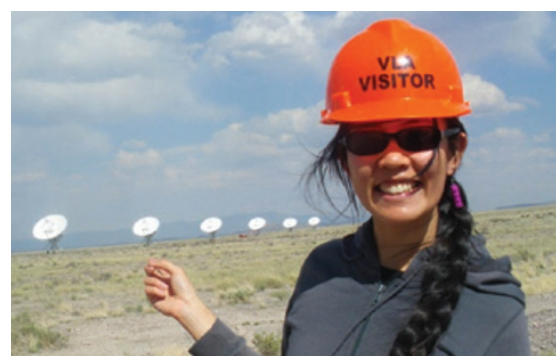

Minnie Mao believes in pushing a

telescope's capabilities.

You asked if I'd given any thought to good strategies for making my own discoveries. I've thought about this a lot. When I was applying for postdocs at the end of last year I looked for jobs that had a telescope support element. I wanted a job that would let me get to know the telescope. I figure that understanding a telescope's capabilities and limitations will enable me to ask science questions that the telescope is able to answer.

I did my PhD in Australia where I spent a lot of time at the Australia Telescope Compact Array (ATCA). I learned so much by being on-site, things like how much microwave ovens affect observations at $13 \mathrm{~cm}$ (a lot!). Experiences like these enabled me to understand my data intimately and hence distinguish real science from image artefacts and other distractions. As such, I felt like I was able to tailor my projects to make the most of the ATCA's capabilities.

Conversely, the VLA has dedicated telescope operators who observe for you. I wish I could do more of the observing myself though. I think learning is not just about accumulating information; it's also about the process of getting that information. I could go away and read all the how-to manuals about the telescope and its data products, but until I actually see the telescope work, and reduce the data myself, I will not truly understand it.

I feel like I'm at the tail-end of the generation of astronomers that gets to actually play with telescopes. Hands-on observing is fast becoming obsolete. What will happen to the next generation of astronomers who write proposals for telescope time? We're always going to have people churning out good, solid science. But is anyone going to have the expertise to come up with something truly innovative?

Unless you are directly involved in the building and commissioning of a new telescope, do you think you can really understand it enough to design creative experiments that will produce groundbreaking science?

\section{Clear skies,}

Minnie

\section{"The challenge is to use our imagination to the fullest."}

\section{Dear Minnie,}

I like your strategy of 'playing with telescopes' to understand their capabilities and limitations. I think it's important because so many great discoveries happen at the very edge of capabilities. The amazing story of the early galaxies is only barely discernible from tiny coloured dots on the Hubble deepfield images, and it has taken tremendous persistence on the part of the observers and instrument builders to know how to: (a) get scientific discoveries from little coloured dots; and (b) get better equipment to see them more clearly. Similarly, what we know of exoplanets has come mostly from people who knew how to push the capabilities of existing equipment (first), and then pushed to get better equipment (after the richness of the discoveries became apparent).

You seem a bit concerned that you won't be able to learn enough about telescopes without touching them. I have that feeling too, but I think we are more and more able to experience things we can't see or touch. After all, astronomy is all about things we can only see from immense distances! I'm writing this on a computer running on hardware I've never seen, based on quantum mechanics that hardly anyone understands. At NASA, I'm working on a giant telescope that for years existed only in the imagination, along with a few documents and viewgraphs and now two of the four instruments are delivered and all of the mirrors are finished.

So, the challenge is to use our imagination to the fullest! Hands-on observing is a way to build that imagination, but total immersion in using something you can't touch still works. So to answer your question, yes, I truly expect there will be many people with the expertise to come up with something truly innovative. We are building the JWST so that a generation of people who were not even born when it was started will be able to use it for breathtaking discoveries.

Fear not, just dive in!

John 


\section{"What I do object to is the complete removal of the astronomer from the observing and data- reduction process."}

\section{Dear John,}

I'm really pleased to hear that you're not worried about the reduction in hands-on observing opportunities. While hands-on observing is something I enjoy passionately, I guess I can understand that it's not necessarily vital to being a brilliant astronomer.

Having said that, I disagree that imagination can replace the wealth of knowledge garnered from sitting in front of a terminal and actually being in control of a telescope. You say "total immersion in using something you can't touch still works", but is that really true? To use your computer analogy, you can read hundreds of books about computers, but you learn most about how to use your computer by sitting at it and actually playing with it.

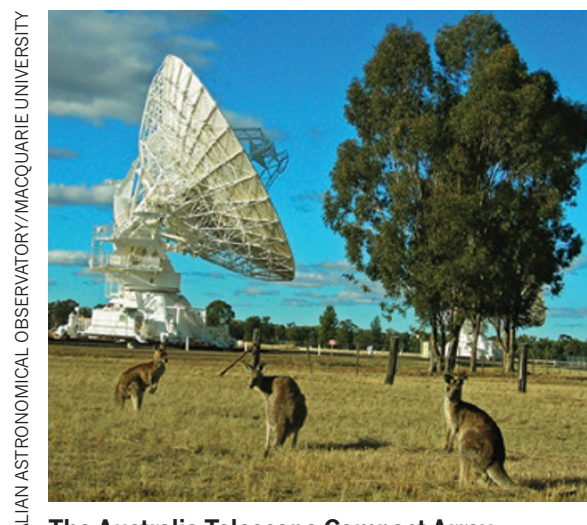

The Australia Telescope Compact Array.

In my last letter, I focused on being physically at a telescope to use it. Obviously this is not feasible for telescopes such as Hubble and the JWST. But even in these circumstances, what I do object to is the complete removal of the astronomer from the observing and data reduction process. Radio telescopes are moving towards a model where the astronomer puts in their observing request and is then provided with completely reduced data. I understand that this is somewhat necessary due to the exorbitantly large sizes of the data sets, but I still find it troubling.

Twenty years ago if you wanted to use a telescope, you went to the telescope, you observed, and you were responsible for reducing your data and analysing it. Nowadays, hands-on observing is becoming less prevalent. Science isn't suffering yet because the astronomers still retain their observing and data-reduction experience. But what happens 20 years from now, with a new generation of astronomers? I'm not convinced that truly innovative ways of exploiting a telescope will be possible when the telescope users are oblivious to the observation and data reduction processes.

What are you planning on doing with the JWST? Will approved observing programs deliver completely reduced data? Will the astronomer even know when their proposed objects are being observed?

Clear skies,

Minnie

\section{"There is never the final answer to anything, only the best answer we think we have at the time."}

\section{Dear Minnie,}

I agree completely that hands-on observing is the way to go when possible. There's nothing like actually touching things and studying them in person to get acquainted. One wouldn't (I hope) marry somebody over the internet without meeting him/her! But as time goes on we all get used to ignoring the inner workings of things, as long as they are reliable. Hardly anyone even knows how a car works these days, or even suspects that it has perhaps a hundred microprocessors running little dedicated tasks. So we get to concentrate on top-level issues, like where to go and how fast. I think modern astronomy has gotten to that point too - we have software packages with millions of lines of code, and we trust that somebody has made them work right. And we have observatories with complexities that are completely hidden from the users most of the time. Of course, the sceptical and cautious user (a real scientist) will not believe everything in the users' manual for either a telescope or a software package, and will find a way to test every important feature, otherwise we will have a lot of non-science. I think the real breakthrough science will still come from people who push the capabilities of these tools beyond their design limits and test what happens. That will take familiarity with design, and imagination to picture what cannot be seen.

About JWST, there's no such thing as completely reduced data from any observatory. We do expect that the observers will receive data already converted into units of astronomical
DNATURE.COM See Mather debating The Golden Age of Astronomy go.nature.com/Luk8pf brightness, according to the best calibration information and algorithms we have. But if an object is very faint, it will have to be observed over a long period of time with multiple exposures that have to be combined; the process of doing the combination is not so simple, and the cautious observer may want to do it him/ herself. But that's still a very long jump from data gathering to interpretation of the objects way out there - that remains the observer's remit. And there is never the final answer to anything, only the best answer we think we have at the time. And yes, the astronomers will know when their proposed objects will be observed. (Small caveat: we are setting up the telescope to have a little autonomy, so if an observation cannot be completed in sequence, the telescope will automatically go to the next one on its list.)

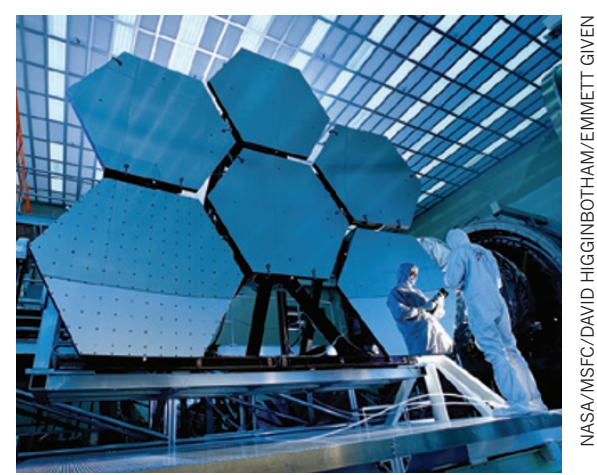

The recently finished mirrors of the James Webb Space Telescope.

I find it very exciting to know of the discoveries made by people working far from my own specialties. And once in a while a conversation occurs that starts a new field of research, just from asking a question about something that is obvious to one person but not to another. We have dark matter in astronomy that is a total mystery. But there is a form of dark matter in genetic material too, and just lately it was announced that it is extremely important: the dark matter between the genes is actually millions of digital switches. I don't know when a breakthrough like that for dark matter and dark energy will occur for astronomers. But why not? And in another area of astronomy, suddenly we know that there are as many exoplanets as there are stars, that small ones are very abundant, and that many have been expelled into space and have no star any more.

So I think one of the most interesting ways to phrase a question in science is: "If X happens, then what?" What do you think are interesting questions?

\section{All the best,}

John

To see this conversation continue online, visit lindau.nature.com/lindau/online dialogue 